\title{
Status of the SK-Gd project
}

\author{
Lluís Martí Magro ${ }^{a, *}$ \\ ${ }^{a}$ University Autónoma Madrid, Department of Theoretical Physics, \\ 28049 Madrid, Spain. \\ E-mail: lluis.marti@uam.es, martillu@km.icrr.u-tokyo.ac.jp
}

Super-Kamiokande (SK) is a 50 kton water Cherenkov detector located approximately $1 \mathrm{~km}$ beneath mount Ikenoyama, Gifu, Japan. While SK can reconstruct charged particle tracks over a wide energy range, the detection efficiency of neutrons is very low. Achieving efficient neutron tagging is useful in all analyses, from the observation of the diffuse supernova neutrino background for the first time, to proton decay studies and oscillation analyses.

SK gadolinium (SK-Gd) is the upgrade project to make neutron tagging efficient. After extensive studies, the SK collaboration approved the SK-Gd project on June 27 2015. In the second half of 2018 we refurbished the detector and in the first half of 2020 we added gadolinium to the SK tank for the first time. Here we briefly report the preparations that led to SK-Gd and then, more extensively, the Gd sulfate loading process.

40th International Conference on High Energy physics - ICHEP2020

July 28 - August 6, 2020

Prague, Czech Republic (virtual meeting)

\footnotetext{
${ }^{*}$ Speaker
} 


\section{Introduction}

Super-Kamiokande (SK) has been running since 1996. Through the years, there have been many improvements and it has undergone several phases. In 2003 the current upgrade started being prepared. Identifying the low efficiency detecting neutron captures in SK as an important handicap for many analyses and suggesting a solution for it, GADZOOKS! was proposed [1]: enrich the otherwise ultra-pure water of SK with a water soluble gadolinium (Gd) salt. Naturally occurring Gd has the largest thermal neutron cross section. This capture produces a gamma cascade of about 8 $\mathrm{MeV}$ which is much easier to detect than the single $2.2 \mathrm{MeV}$ gamma produced after a neutron capture on hydrogen. With the carrot of high neutron capture detection efficiency in front of us, a large scale project (EGADS for Evaluating Gadolinium's Action on Detector Systems) started to test the feasibility of this idea. Since then many studies were conducted to answer many questions around this technique. For gadolinium sulfate (the best salt tested), can we add it and remove it (if needed) in an efficient/economical way? How does it affect the water transparency? Can the purification system maintain a good water quality? Are there adverse effects on detector components? Can we reduce the neutron background low so as not to negatively affect current analyses?. These questions were addressed in a recent paper [2] where some of the benefits were described as well.

These benefits range from supernova ( $\mathrm{SN}$ ) neutrinos; with improved pointing accuracy, enhanced sensitivity to late black hole formation and detection of stellar neutrinos fusing silicon for nearby stars just before going SN, or the search of the diffuse SN neutrino background from all the $\mathrm{SNe}$ in the history of the universe; to sensitivity gains in proton decay searches.

Because of all the benefits and the positive results from EGADS about this technique, it was decided to implement it in SK. The first step was to refurbish the SK detector in 2018. Among others, the detector walls and structures were cleaned and faulty inner and outer photomultipliers were replaced. A new hall had been excavated and a new water purification system installed. This system can remove all impurities in water but keep Gd dissolved. The in-tank piping was also modified in order to be able to increase the total flow (increasing the water purification power) and allow for an independent flow control between the inner and the outer detector. The previously existing water leak was reduced to an undetectable level (less than $20 \mathrm{~L} /$ day) by sealing the weld plates and penetrating bolts of the detector.

$\mathrm{Gd}$ sulfate, figure 1a, is very easy to dissolve when octahydrated, $\mathrm{Gd}_{2}\left(\mathrm{SO}_{4}\right)_{3} \cdot 8 \mathrm{H}_{2} \mathrm{O}$ (hereafter, referred as $\mathrm{Gd}$ sulfate). A concentration of $0.02 \%$ in weight is enough to achieve $50 \%$ of the neutrons captures on $\mathrm{Gd}$, figure $1 \mathrm{~b}$. This concentration was our first goal.

$\mathrm{Gd}$ sulfate was first dissolved, already with the final concentration of $0.02 \%$ in weight, and then injected into SK from the bottom, figure 2. Laminar flow in SK is achieved through flow and temperature control. The goal was to create two separated regions: a Gd sulfate loaded region and a pure water region. Pure water was drawn from the top, Gd sulfate was dissolved and then injected back into SK from the bottom until the SK tank was fully loaded. During injection, water was sampled directly from the SK tank at different depths and from different sampling ports. The water conductivity (which in very pure water tracks Gd sulfate) and the Gd sulfate concentration were measured from these samples to trace the injection process. The results, figure 3 , show that there was a clear separation between the Gd sulfate loaded and the pure water regions (very vertical distributions in the boundary) with a 2 meter layer separation between them. The Gd sulfate region 

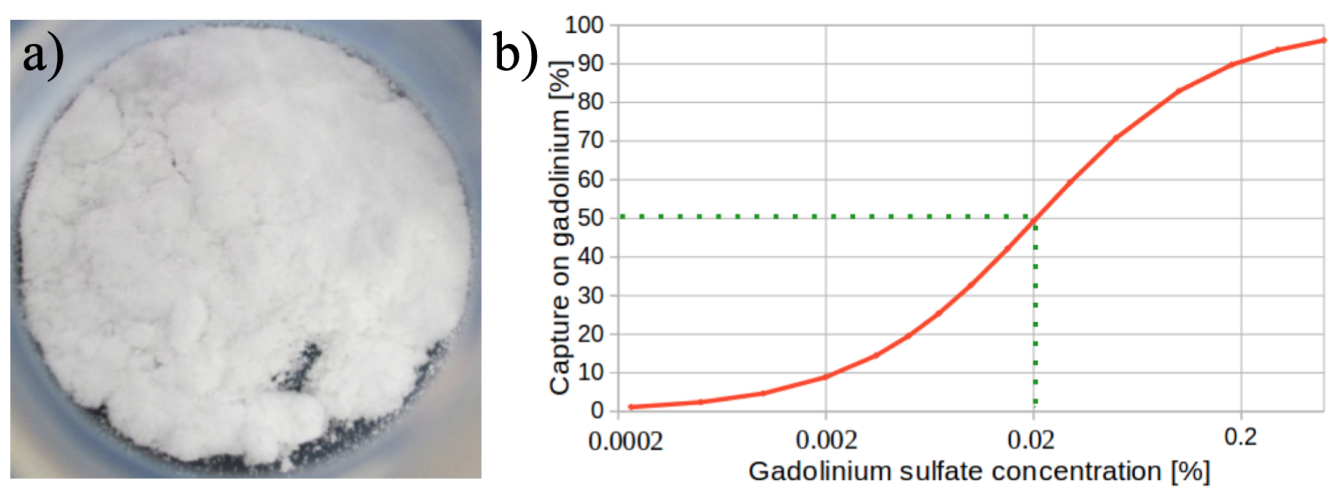

Figure 1: Octahydrated gadolinium sulfate (a) and thermal neutron capture [\%] as a function of the concentration in [\%] weight of octahydrated gadolinium sulfate in water (b). The target concentration for the current loading, $0.02 \%$ (about $50 \%$ of neutron captures) is indicated.

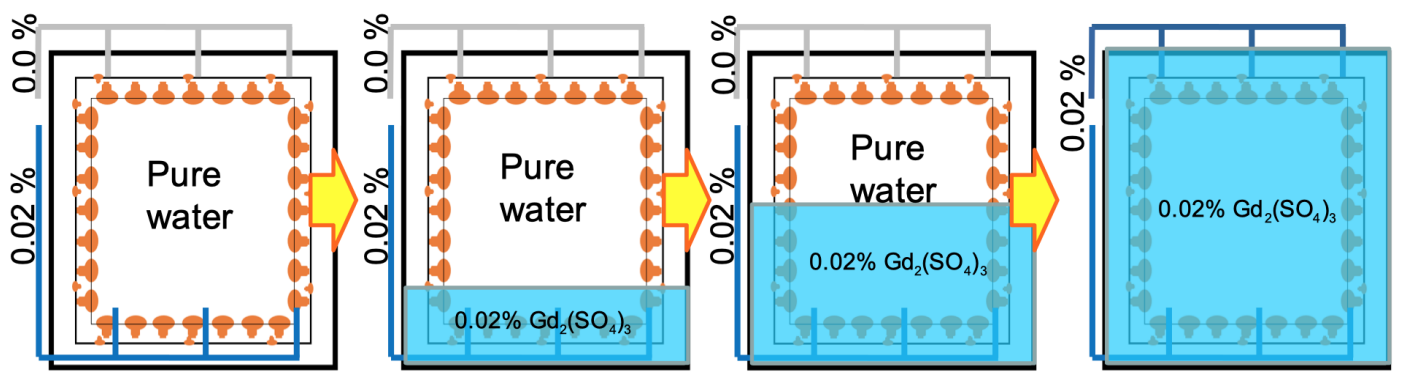

Figure 2: Pure water is drawn from the top, Gd sulfate is dissolved and then injected back into the SK tank from the bottom.

advanced upwards at a speed of about 1.5 meter per day.

Several neutron sources can be used to both track the Gd loading process and determine the $\mathrm{Gd}$ sulfate concentration as well. Using these sources during the Gd loading process, we searched for the first indications that we could indeed now efficiently detect neutrons. Here we will show some examples while the Gd sulfate was still well below the lower half of the detector.

One of these sources is an americium-beryllium $(\mathrm{Am} / \mathrm{Be})$ source inside a bismuth germanate (BGO) crystal. Beryllium atoms capture alpha particles produced by ${ }^{241} \mathrm{Am}$ which yields a neutron and a 4.4 MeV gamma. These gammas are then converted to scintillation light by the BGO crystal while the neutron can then be captured outside the crystal by Gd. We looked for neutron capture candidate events after each prompt event triggered by the scintillation light. The variable $\mathrm{Z}$ is the vertical distance from the center of the tank, being zero at the center, negative below and positive above it. We deployed the $\mathrm{Am} / \mathrm{Be}$ source at three positions: bottom ( $\mathrm{Z}=-12$ meters), center ( $\mathrm{Z}=0$ meters) and top ( $Z=+12$ meters) of the tank. Figure 4 (left) shows the reconstructed energy of these events. Only for those events when the source is at the bottom position, the energy is compatible with neutron captures on $\mathrm{Gd}$, around $5 \mathrm{MeV}$ (about the expected Cherenkov light energy equivalent for the $8 \mathrm{MeV}$ neutron capture gamma cascades). On the other hand, for the other positions, the energy is compatible with captures on hydrogen. Due to the low efficiency at $2.2 \mathrm{MeV}$ we see the peak of these neutron captures around $3.5 \mathrm{MeV}$. Figure 4 (right) shows the capture time of 


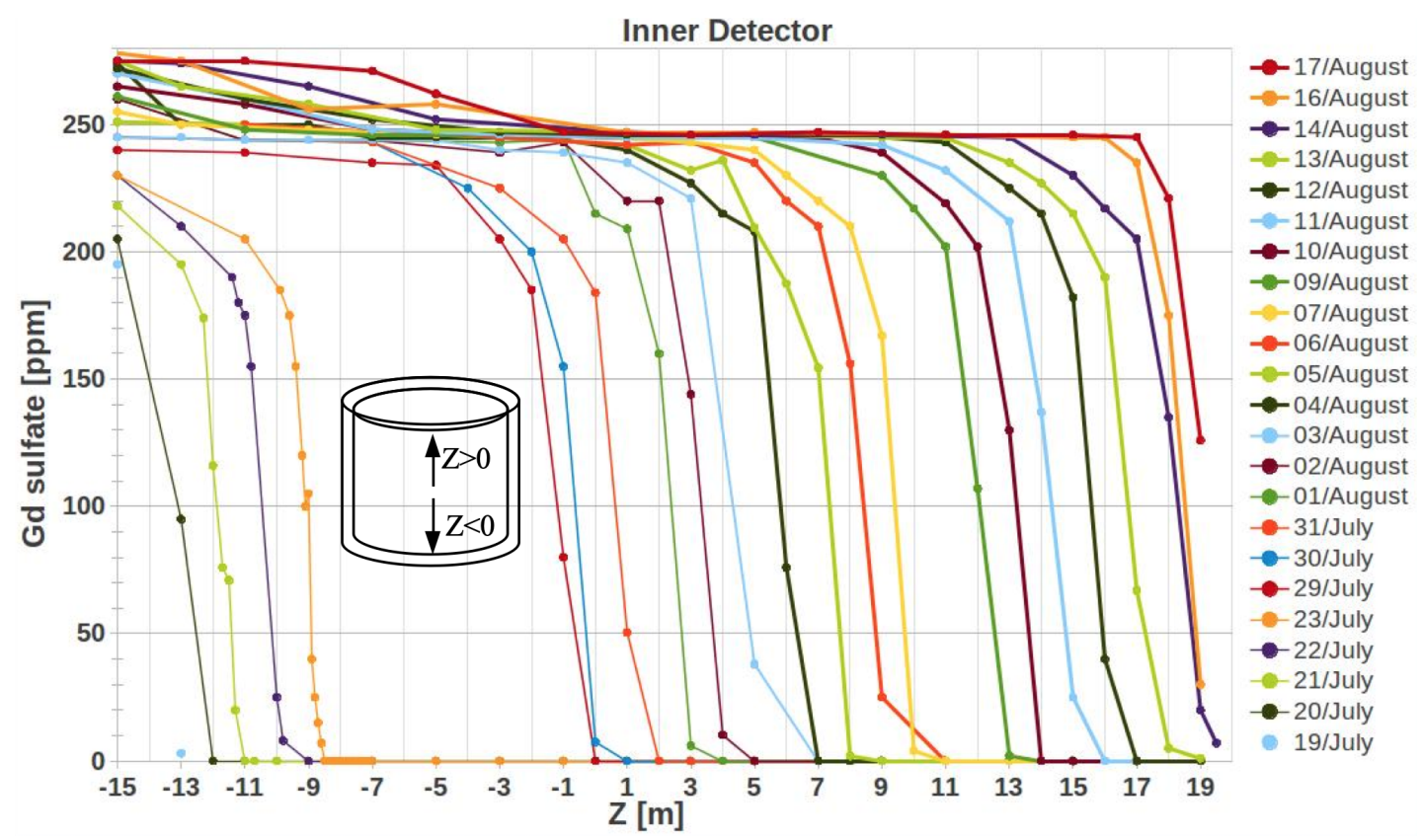

Figure 3: Day by day Gd sulfate concentration at several depths in the SK tank $(Z=0$ being the center of the tank).

the neutron candidates at $\mathrm{Z}=-12$ meters, i.e. the time difference between the prompt event, the scintillation light, and the neutron capture candidate. The fitted time constant, $132 \pm 5 \mu \mathrm{s}$, is also compatible with our Monte Carlo (MC) simulations with the target concentration.

At SK we observe cosmic ray muons with an average rate of about $2 \mathrm{~Hz}$. These muons can produce spallation neutrons. See cartoon in figure 5 (left). The number of produced spallation neutrons is of the order of the tens of thousands every day. We searched for parent muons in the lower region of the detector $(Z<0)$ and then for neutron candidates around them. In figure 5, a run with gadolinium and another one without are compared. Figure 5 (left) shows the reconstructed energy of the neutron captures. As before, the neutron captures on Gd are in the expected region around $5 \mathrm{MeV}$ for the run with $\mathrm{Gd}$ but not in the run before Gd injecton. On the right, we show the time of neutron capture candidates. The fitted time constant is $136 \pm 3 \mu s$ which is in agreement with both the MC simulation and the previous method. For the run without $\mathrm{Gd}$, a rather flat distribution is observed, which is also expected in this case.

Sometimes a cosmic muon stops in our detector and is captured on an oxygen atom. Candidates are stopping muon tracks. Then transforms into a nitrogen atom and later a neutron is kicked out the nucleus, see cartoon in figure 6 . We search for neutron captures on $\mathrm{Gd}$ at different slides in $\mathrm{Z}$ in our detector. Here, four regions are shown: $-16 \mathrm{~m}<\mathrm{Z}<-12 \mathrm{~m},-12 \mathrm{~m}<\mathrm{Z}<8 \mathrm{~m},-8<\mathrm{Z}<-4$ and $-4 \mathrm{~m}<\mathrm{Z}<0$, upper left, lower left, upper right and lower right respectively figure 6 . Since we inject Gd sulfate from the bottom, we expect to see neutron captures in the lowest regions earlier than in the upper ones. In the bottom most region $(-16 \mathrm{~m}<\mathrm{Z}<-12 \mathrm{~m})$ we observe the first neutron captures before any other region in the inner detector. A delay between the injection start and the first observed captures in the lower region of the inner detector can be seen. This is because the bottom outer detector had to be filled with Gd sulfate first. For the other regions, as expected, longer 

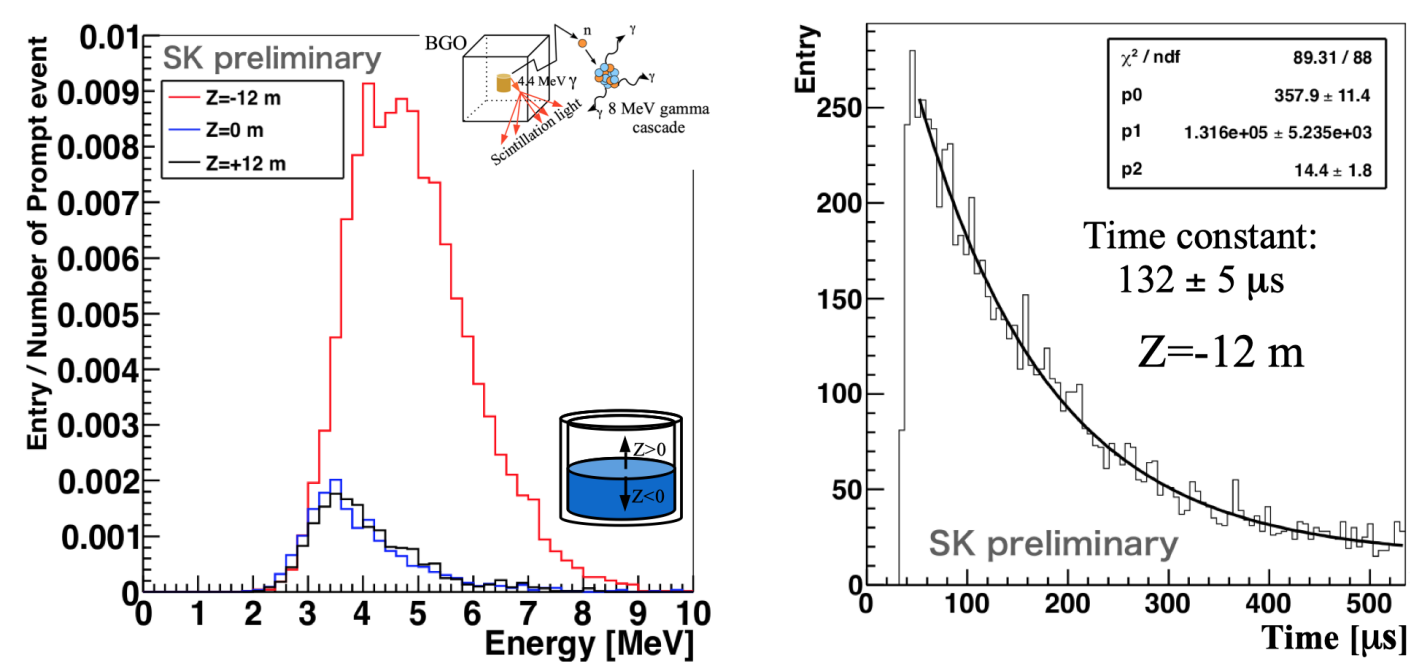

Figure 4: Reconstructed energy for neutron capture candidate events after each prompt event (triggered by the BGO scintillation light) when deploying the $\mathrm{Am} / \mathrm{Be}$ source at three positions: bottom $(\mathrm{Z}=-12$ meters $)$, center ( $\mathrm{Z}=0$ meters) and top $(\mathrm{Z}=+12$ meters) of the tank (left). Capture time of the neutron candidates at $\mathrm{Z}=-12$ meters (right).
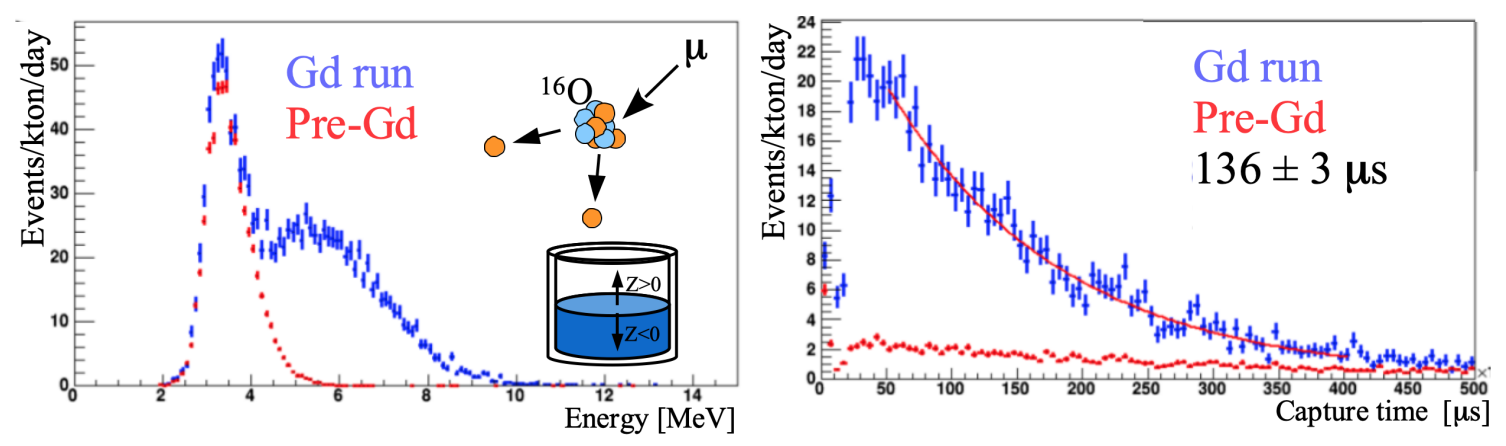

Figure 5: Reconstructed energy for spallation neutrons candidates for runs with and without Gd in the lower region of the detector (left) and capture time of the neutron candidates (right).

time is needed for Gd to reach them and this is what is observed.

On August 17th the Gd sulfate loading was finished. Similar to the tests described above, and other tests as well, have been conducted during and after the Gd sulfate injection. One of the most important things to check after this process was to check the homogeneity of the Gd sulfate concentration in SK. Figure 7 shows the results of concentration measurements from samples taken after the injection. By September 8th we already observed a very homogeneous concentration across the whole detector. Further samples have been taken later (not shown for clarity). We observed that the concentration homogeneity further improved in the detector with concentration differences well below $1 \%$.

More details of the Gd sulfate loading and related studies are expected to be published soon. Extensive studies in the detector performance are still underway and will be reported too in the future. A new era has started now for SK with an efficient neutron tagging which will improve current analyses and make possible new discoveries. We are also preparing for the next stage of Gd 

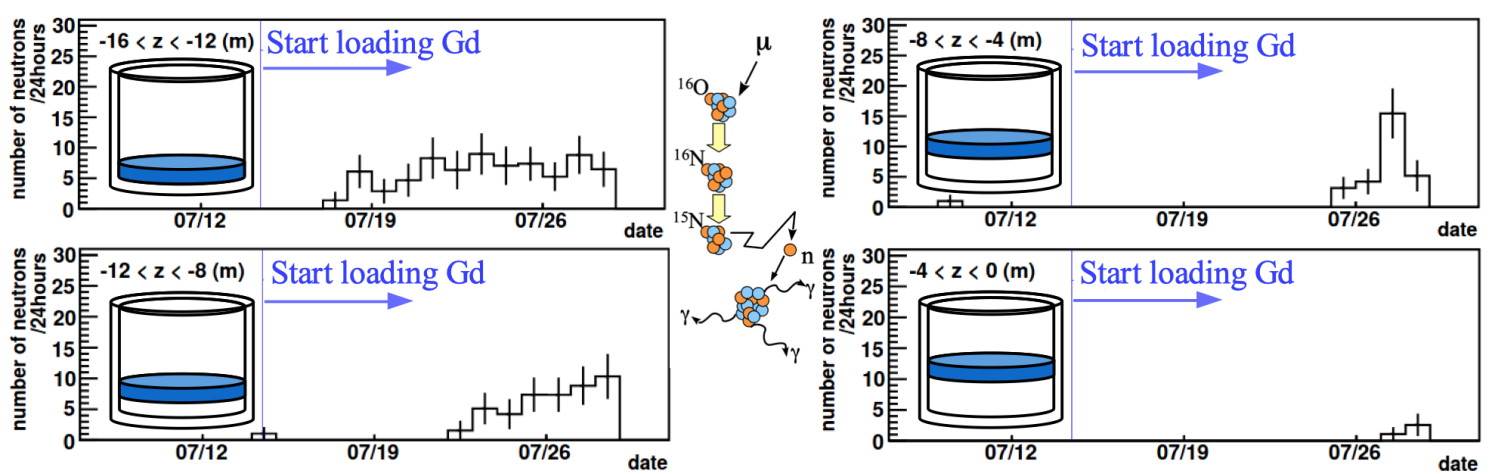

Figure 6: Neutron captures from stopping muons in four different regions $(-16 \mathrm{~m}<\mathrm{Z}<-12 \mathrm{~m},-12 \mathrm{~m}<\mathrm{Z}$ $<8 \mathrm{~m},-8<\mathrm{Z}<-4$ and $-4 \mathrm{~m}<\mathrm{Z}<0$, upper left, lower left, upper right and lower right respectively) while loading Gd sulfate in SK.

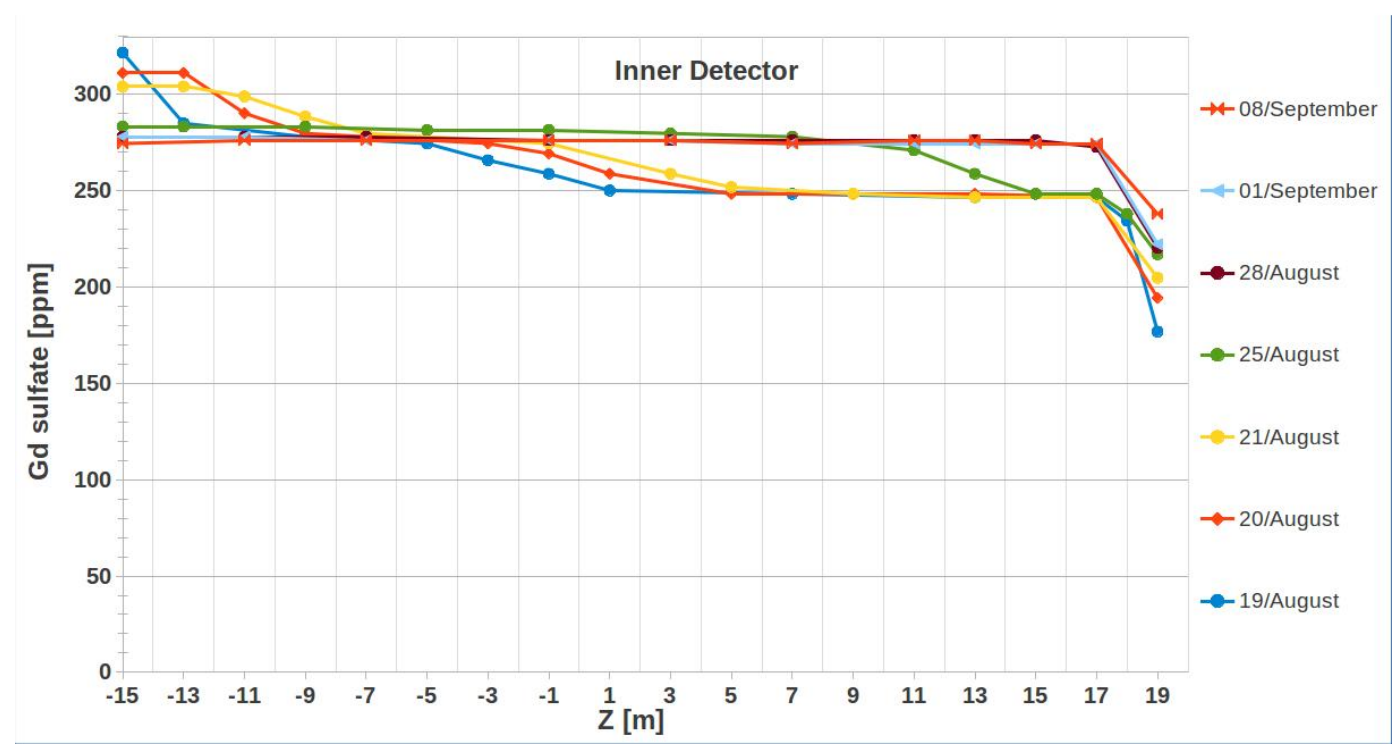

Figure 7: Gd sulfate concentration after finishing Gd sulfate injection. The concentration becomes more and more homogeneous in the whole SK tank.

loading.

\section{References}

[1] Beacom, John F. and Vagins, Mark R., GADZOOKS! Anti-neutrino spectroscopy with large water Cherenkov detectors", Phys. Rev. Lett., 93, 171101, 2004.

[2] Marti, Ll. and others, Evaluation of gadolinium's action on water Cherenkov detector systems with EGADS, Nucl. Instrum. Meth. A, 959, 163549, 2020. 